\title{
Matrine improved the function of heart failure in rats via inhibiting apoptosis and blocking $\beta 3$-adrenoreceptor/endothelial nitric oxide synthase pathway
}

\author{
JIANGBO YU, SHUSEN YANG, XU WANG and RUNTAO GAN
}

Department of Cardiology, The First Affiliated Hospital of Harbin Medical University, Harbin, Heilongjiang 150001, P.R. China

Received December 3, 2013; Accepted June 19, 2014

DOI: $10.3892 / \mathrm{mmr} .2014 .2642$

\begin{abstract}
Matrine, an alkaloid isolated from the traditional Chinese medicine Sophora flavescens AIT has exhibited a number of therapeutic effects on cardiovascular and liver diseases. The purpose of the present study was to investigate whether matrine has a protective effect on heart failure in rats. Coronary artery ligation was used to induce a heart failure (CHF) model in rats. Four weeks following the procedure, the rats were treated with different doses of matrine for one month. Histopathological examination demonstrated that matrine treatment alleviated myocardial hypertrophy and cardiac fibrosis in failing hearts. Furthermore, matrine administration also inhibited the increase of plasma aspartate amino transferase, creatine phosphokinase and lactate dehydrogenase levels in CHF rats. The rats with heart failure exhibited a significant reduction in ejection fraction and fractional shortening, as well as an increase in the left ventricular end systolic dimension, and matrine attenuated this decline in heart function. Further investigation demonstrated that matrine treatment also inhibited the upregulation of Bax and increase in the Bcl-2 expression in the failing hearts. Furthermore, the upregulation of $\beta 3$-adrenoreceptor (AR) and endothelial nitric oxide synthase proteins following heart failure were also attenuated by matrine. In conclusion, matrine had a preventive role in heart failure in rats at least in part by inhibiting myocardial apoptosis and the $\beta 3$-AR pathway.
\end{abstract}

\section{Introduction}

As a common traditional Chinese medicine, Sophora flavescens AIT has been used to treat tumors, viral hepatitis, viral myocarditis, skin diseases and arrhythmia (1-2). The active compounds of Sophora flavescens AIT consist of alkaloids, flavones and aralosides (2). As a major quinolizindine alkaloid isolated

Correspondence to: Professor Runtao Gan, Department of Cardiology, The First Affiliated Hospital of Harbin Medical University, Internal Medicine Building, 23 Youzheng Street, Harbin, Heilongjiang 150001, P.R. China

Email: yujiangbohmu@163.com; ganrt@126.com

Key words: matrine, heart failure, apoptosis from Sophora flavescens AIT, matrine possesses antitumor, antivirus, antioxidant and anti-inflammatory actions (1,3-5). The anticancer effects of matrine were reported in murine hepatocellular carcinoma cells via regulating Bcl-2 and Bax expression (1). Matrine was also demonstrated to activate the p38 pathway and trigger caspase-dependent apoptosis by inducing the generation of ROS in non-small cell lung carcinoma (6). Matrine is able to inhibit cell proliferation and induce the apoptosis of acute myeloid leukemia cells via the mitochondrial pathway and Akt inactivation (7). Furthermore, the growth of MCF-7 breast cancer cells was also inhibited by matrine via the miR-21/PTEN/Akt pathway (8). Additionally, matrine exhibited antinociceptive effects on neuropathic pain induced by chronic constriction injury in mice (9). Matrine was also effective in preventing the conversion of high-fructose diet-induced hepatic steatosis into non-alcoholic steatohepatitis in rats via regulating Nrf2 (5).

It was also reported that matrine had a protective role in cardiac conditions $(10,11)$. In one study, matrine triggered the apoptosis of cardiac fibroblasts and thereby inhibited Ang II-induced hyperplasic growth via the regulation of Bcl-2/Bax expression and caspase-3 activation (11). Atrial fibrillation was also prevented by matrine through the downregulation of $\mathrm{I}_{\mathrm{KM} 3}$ density and upregulation of $\mathrm{ICa}-\mathrm{L}$ density (12). In addition, matrine produced a positive inotropic effect on rat hearts through enhancing the calcium influx (13). Although a series of studies demonstrated that matrine exerted numerous protective effects on liver, heart, vessels and other tissues $(1,2,4,5,9-12)$, whether matrine is capable of treating heart failure, and the potential mechanism underlying this effect remain unknown (14). Therefore, it was hypothesized that matrine may produce therapeutic effects on heart failure. To examine this hypothesis, the present study was designed to observe the protective role of matrine in failing hearts and investigate its molecular mechanisms.

\section{Materials and methods}

Animals. Wistar rats (weight, 230-300 g) were purchased from the Experimental Animal Center of the First Affiliated Hospital of Harbin Medical University (Harbin, China), and were housed in plastic cages on aspen-chip bedding under conditions of controlled temperature $\left(18-21^{\circ} \mathrm{C}\right)$ and humidity 
$(55 \pm 5 \%)$ with a $12 / 12 \mathrm{~h}$ light/dark cycle. All of the experimental procedures performed on the animals were in accordance with the guidelines of the Animal Ethics Committee of Harbin Medical University (no. 20130013, Jan 15th, 2012).

Drugs. Matrine (purity, $>98 \%$ via high performance liquid chromatography) was purchased by Shaanxi Huike Botanical Development Co., Ltd (Xi'an, China), and was dissolved in $0.9 \%$ sodium chloride saline solution in all of the animal experiments. All of the other reagents were purchased from Sigma-Aldrich (St. Louis, MO, USA) unless otherwise specified.

Establishment of heart failure. Wistar rats were randomly divided into sham operation and heart failure groups. The method to establish a heart failure model in rats using coronary artery ligation was performed as described previously, with certain modifications (15). Briefly, the rat chest was opened and the heart was exposed. The left anterior descending coronary artery was occluded with a black braided silk suture (Ethibond; Johnson \& Johnson, New Brunswick, NJ, USA) $\sim 2 \mathrm{~mm}$ below its origin. The ligature was placed but was not tied in the sham-operated animals. Four weeks following the procedure, ejection fraction (EF) values ranging from $30-55 \%$ (heart failure) were included in the further studies. Heart failure groups were further divided into: $3 \mathrm{mg} / \mathrm{kg}$ matrine, $10 \mathrm{mg} / \mathrm{kg}$ matrine, $30 \mathrm{mg} / \mathrm{kg}$ matrine and saline control groups. Matrine was administered to the rats for four consecutive weeks.

Histopathology. All of the rats were sacrificed by exsanguination under anesthesia. The heart tissues were sectioned into a 5-mm-thick cross-section at the level of papillary muscle, fixed in $10 \%$ formalin, dehydrated and embedded in paraffin. The slices from the section underwent hematoxylin-eosin (H\&E) and Masson's trichrome staining. Ten visual fields were randomly selected in each slice stained with Masson's trichrome under a microscope (Olympus AX80; Olympus Corporation, Tokyo, Japan) and measured by a computer-assisted image analysis system (Motic Med 6.0; CMIAS, Beijing, China).

Echocardiography. Following being anesthetized with $30 \mathrm{mg} / \mathrm{kg}$ pentobarbital sodium, echocardiography was performed in rats with a $12 \mathrm{MHz}$ phased-array SONOS-7500 transducer (Philips, Eindhoven, The Netherlands). Two dimensional parasternal long axis views and short axis views were obtained at the papillary muscle level. Left ventricular end diastolic dimension (LVEDD) and left ventricular end systolic dimension (LVEDS) were measured. The ejection fraction and fractional shortening were then calculated. All of the measurements were averaged from three consecutive heart cycles and were performed by an experienced technician.

Measurement of aspartate amino transferase (AST), creatine phosphokinase $(C P K)$ and lactate dehydrogenase $(L D H)$ levels. The levels of plasma AST, LDH and CPK were measured using AST, LDH and CPK kits supplied from Beijing Zhongsheng Biological Co., Ltd (Beijing, China) according to the manufacturer's instructions. The results are expressed as the IU/l for AST, LDH and CPK.
Western blot analysis. Total proteins were isolated from the cardiac tissue and the concentration was determined using the Bradford method (Bio-Rad Laboratories, Hercules, CA, USA). The crude protein extracts $(30-50 \mu \mathrm{g})$ were then loaded onto a 10-12\% SDS-poly-acrylamide gel and transferred to nitrocellulose membranes. The membranes were blocked for $1 \mathrm{~h}$ in phosphate-buffered saline with Tween-20 with 5\% non-fat milk and probed with primary monoclonal anti- $\beta$-actin, polyclonal anti-Bcl-2, monoclonal anti-Bax, polyclonal anti$\beta 3$-adrenoreceptor (AR) and polyclonal anti-endothelial nitric oxide (eNOS) antibodies (Santa Cruz Biotechnology, Inc., Santa Cruz, CA, USA). Following incubation with a horseradish peroxidase-conjugated goat anti-mouse monoclonal secondary antibody for $1 \mathrm{~h}$ (Sigma-Aldrich), the bands on the blots was visualized by Western blue (Promega Corporation, Madison, WI, USA) and quantified using the computer image analysis system (Image J; National Institutes of Health, Bethesda, MA, USA). The expression level of eNOS and $\beta 3$-AR were normalized by comparison with $\beta$-actin.

Statistical analysis. The group data are expressed as the mean \pm standard error of the mean. Analysis of variance was used to compare the group data from the animal experiments. Statistical calculations were performed on a personal computer using SPSS 13.0 for Windows (SPSS Inc., Chicago, IL, USA) . A two-tailed $\mathrm{P}<0.05$ was considered to indicate a statistically significant difference.

\section{Results}

Protective effects of matrine on the structure of failing hearts. Firstly, a heart failure model was established in rats by coronary artery ligation according to our previous study (15), and then the protective effects of matrine on the functions of failing hearts was observed. As demonstrated in Fig. 1A, left ventricular weight (LVW)/body weight (BW) and heart weight (HW)/body weight (BW) ratios were significantly increased in the CHF group compared with the control group $(n=10$, $\mathrm{P}<0.05$ ). Treatment with matrine 3,10 and $30 \mathrm{mg} / \mathrm{kg}$ evidently inhibited the increase of LVW/BW and HW/BW ratio in failing hearts. Fig. 1B demonstrates that interstitial fibrosis and cardiac hypertrophy were found in the CHF group but not in the control group. Matrine $(10 \mathrm{mg} / \mathrm{kg})$ treatment also alleviated the structural remodeling of the failing hearts.

Effects of matrine on the serum level of AST, LDH and CPK in failing hearts. Next, the effects of matrine on the serum levels of AST, LDH and CPK in rats with heart failure were observed. As demonstrated in Fig. 2, the rats with CHF demonstrated a significant increase in AST, LDH and CPK levels as compared with the control group $(n=10, P<0.05)$. Treatment with 10 and $30 \mathrm{mg} / \mathrm{kg}$ matrine significantly decreased the enhanced AST, $\mathrm{LDH}$ and CPK levels in failing hearts $(\mathrm{n}=10, \mathrm{P}<0.05)$. These results suggest that myocardial injury in failing hearts may be attenuated by matrine treatment.

Matrine improves the cardiac function in rats with heart failure. In the present study, echocardiography was employed to observe the effects of matrine on heart function among the three groups. Fig. 3A reveals the samples of M-mode echocar- 
A
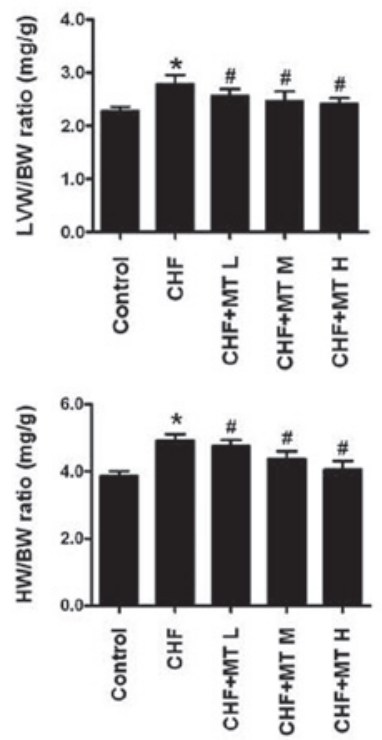

HE

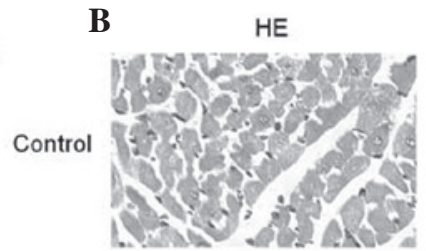

$\mathrm{CHF}$

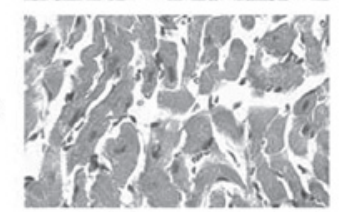

$\mathrm{CHF}+\mathrm{MT} \mathrm{M}$

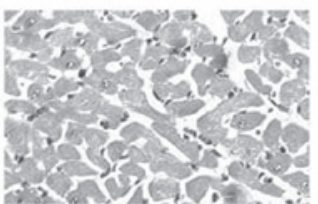

Masson
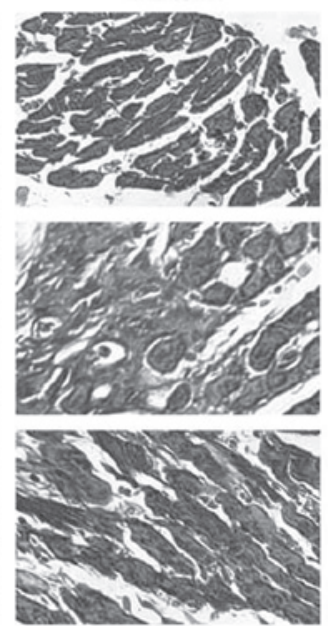

Figure 1. Preventive effects of matrine on structural remodeling in rats with heart failure. (A) Matrine inhibited the increase of LVW/BW and HW/BW ratios in failing hearts. (B) Hematoxylin-eosin staining demonstrated that myocardial hypertrophy and fibrosis were evidently attenuated by $10 \mathrm{mg} / \mathrm{kg}$ matrine in failing hearts (magnification, x200). $\mathrm{n}=10$ per group; MT L, MT M and MT H represent matrine 3, 10 and $30 \mathrm{mg} / \mathrm{kg}$, respectively. "P<0.05 vs. saline control; ${ }^{\#} \mathrm{P}<0.05$ vs. CHF. CHF, coronary artery ligation-induced heart failure; LVW, left ventricular weight; BW, body weight; HW, heart weight.
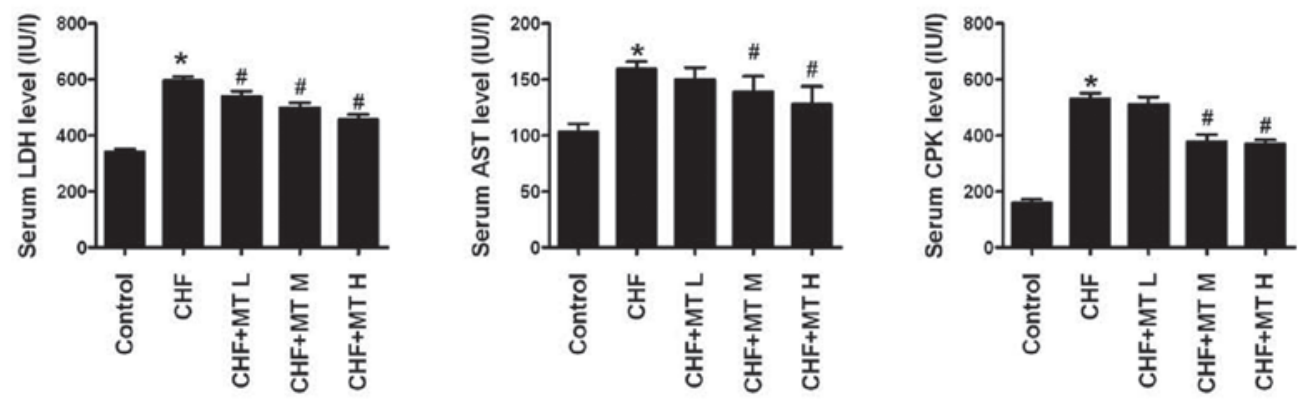

Figure 2. Effects of matrine on biomedical parameters in failing hearts. The serum levels of AST, LDH and CPK were significantly increased in heart failure. However, treatment with matrine (10 and $30 \mathrm{mg} / \mathrm{kg}$ ) significantly inhibited the augmentation of AST, LDH and CPK levels in failing hearts. $\mathrm{n}=10 \mathrm{per}$ group; MT L, MT M and MT H represent matrine 3, 10 and $30 \mathrm{mg} / \mathrm{kg}$, respectively. ${ }^{*} \mathrm{P}<0.05$ vs. saline control; ${ }^{~} \mathrm{P}<0.05$ vs. CHF. CHF, coronary artery ligation-induced heart failure; AST, aspartate amino transferase; CPK, creatine phosphokinase; LDH, lactate dehydrogenase.

A

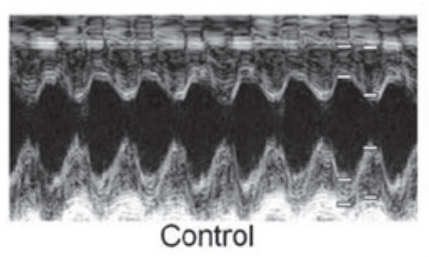

B

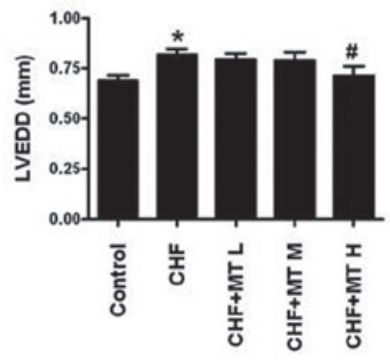

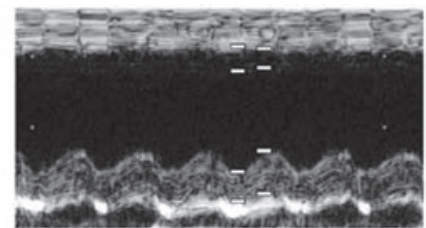

$\mathrm{CHF}$

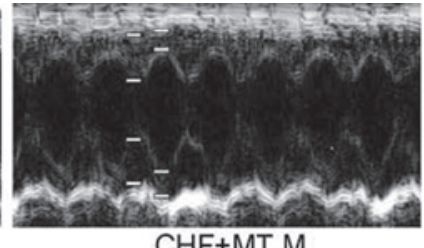

CHF+MT M
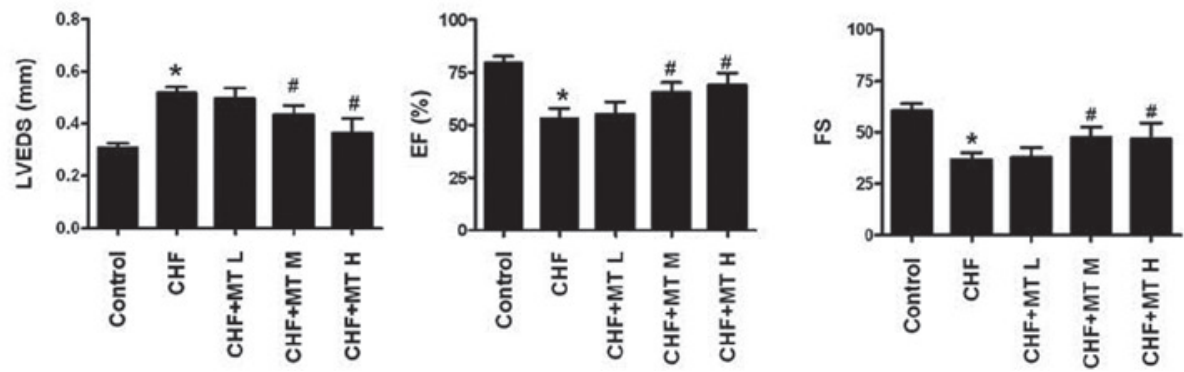

Figure 3. Matrine administration inhibited the cardiac function of failing hearts. (A) The representative echocardiography samples from the hearts of three groups. (B) EF and FS were evidently decreased, and LVEDD and LVEDS were enhanced in rats with heart failure. Matrine treatment (10 and $30 \mathrm{mg} / \mathrm{kg}$ ) inhibited the changes of EF, FS and LVEDS in failing hearts. $\mathrm{n}=10$ per group; MT L, MT M and MT H represent matrine 3,10 and $30 \mathrm{mg} / \mathrm{kg}$, respectively.

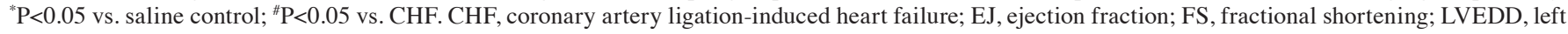
ventricular end diastolic dimension; LVEDS, left ventricular end systolic dimension (LVEDS) were measured. 
A

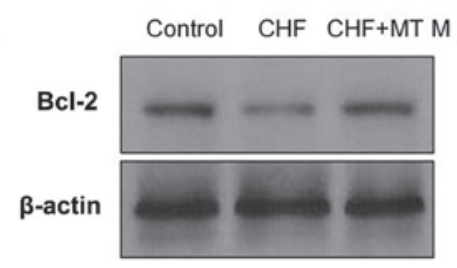

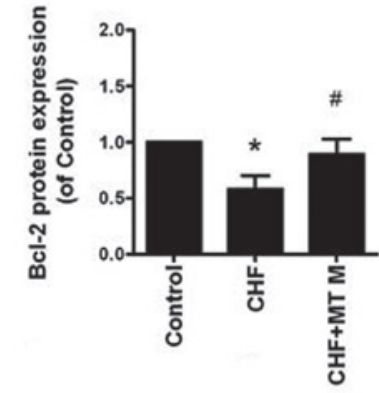

B

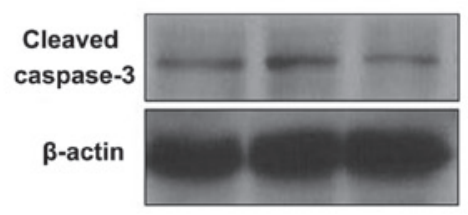

$$
\text { Bax }
$$

$\beta$-actin
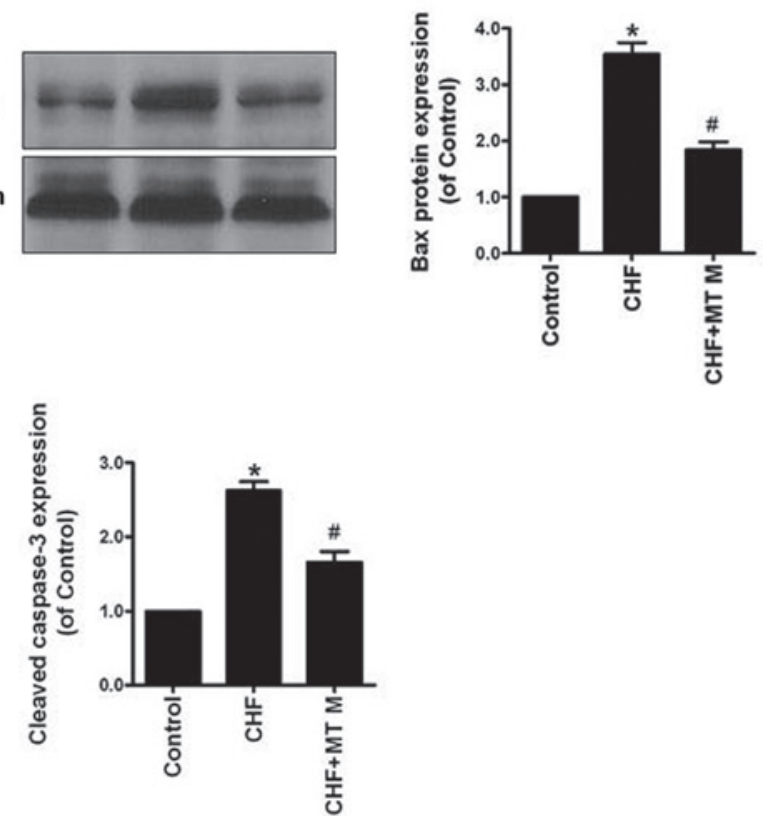

Figure 4. Effects of matrine on the apoptotic proteins in hearts. (A) The expression of Bcl-2 protein was downregulated, and the expression of Bax protein was increased in the ventricles of failing hearts. Matrine treatment $(10 \mathrm{mg} / \mathrm{kg})$ inhibits the downregulation of Bcl-2 and the upregulation of Bax in failing hearts. (B) Matrine reversed the increase of cleaved caspase- 3 protein in failing hearts. $\mathrm{n}=5$ for each group; MT M represents matrine $10 \mathrm{mg} / \mathrm{kg}$. ${ }^{*} \mathrm{P}<0.05 \mathrm{vs}$. saline control; ${ }^{\#} \mathrm{P}<0.05$ vs. CHF. CHF, coronary artery ligation-induced heart failure.

A

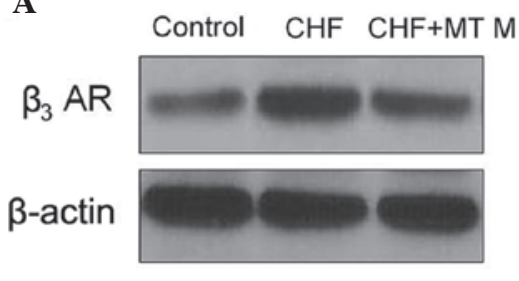

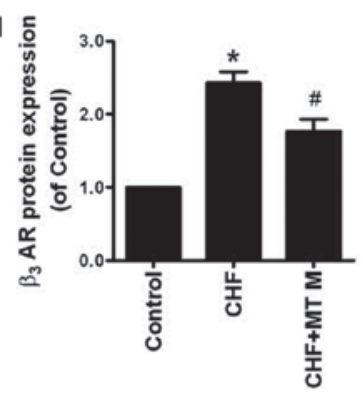

B

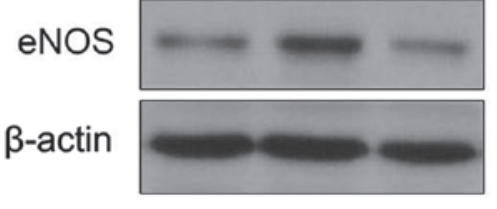

Figure 5. Matrine blocked $\beta 3-\mathrm{AR}$ and eNOS overexpression in failing hearts. (A) The expression of $\beta 3$-AR protein was evidently increased and (B) the eNOS protein was evidently upregulated in rats with heart failure. Following matrine $(10 \mathrm{mg} / \mathrm{kg})$ treatment, the overexpression of $\beta 3$-AR and eNOS proteins was inhibited in failing hearts. $\mathrm{n}=5$ for each group; MT M represents matrine $10 \mathrm{mg} / \mathrm{kg}$. " $\mathrm{P}<0.05$ vs. saline control; " $\mathrm{P}<0.05$ vs. CHF. CHF, coronary artery ligation-induced heart failure; $\beta 3$-AR, $\beta 3$-adrenoreceptor; eNOS, endothelial nitrc oxide.

diograms from the control, $\mathrm{CHF}$ and matrine treatment groups. Echocardiographical examination demonstrated that EF and FS were significantly decreased in the CHF group compared with the control group $(\mathrm{P}<0.05)$, and LVEDD and LVEDS were significantly increased in the CHF group compared with the control group (Fig. 3B). Matrine treatment evidently attenuated the increase in LVEDS, as well as the reduction of EF and FS $(n=10, P<0.05)$. This suggests that matrine administration (10 and $30 \mathrm{mg} / \mathrm{kg}$ ) is able to prevent the decline of cardiac function in rats with heart failure (Fig. 3B).

Matrine inhibits apoptotic proteins in failing hearts. To examine whether apoptosis is involved in the prevention of heart failure by matrine, the effects of matrine on the expression of proapoptotic proteins in failing hearts was examined. As demonstrated in Fig. 4A, it was identified that $10 \mathrm{mg} / \mathrm{kg}$ matrine evidently inhibited the reduction of $\mathrm{Bcl}-2$ protein and the increase of Bax in the CHF group ( $\mathrm{n}=5, \mathrm{P}<0.05)$. The results also revealed that the expression of cleaved caspase-3 protein was increased in the failing hearts compared with the control $(\mathrm{n}=5, \mathrm{P}<0.05)$, and $10 \mathrm{mg} / \mathrm{kg}$ matrine was able to inhibit the activation of cleaved caspase-3 protein in failing hearts (Fig. 4B). This suggests that the inhibition of apoptosis by matrine is involved in its protective effects on heart failure.

Overexpression of $\beta 3-A R$ is attenuated by matrine in heart failure. Our previous study demonstrated that the expression of $\beta 3$-AR and eNOS protein was significantly increased in the failing heart (16). The overexpression of $\beta 3$-AR contributed to the decrease in the contractility of cardiac muscle (16). Therefore, a $\beta 3$-AR antagonist was suggested as a therapeutic drug for heart failure. In the present study the effects of matrine on the activation of the $\beta 3-\mathrm{AR} / \mathrm{eNOS}$ pathway in 
failing hearts were further investigated. Western blot analysis was used to detect the expression of $\beta 3-\mathrm{AR}$ and eNOS protein in the failing hearts. As demonstrated in Fig. 5A, although $\beta 3-\mathrm{AR}$ protein was significantly increased in the $\mathrm{CHF}$ group compared with the control group $(n=5, P<0.05)$, the overexpression of $\beta 3-\mathrm{AR}$ protein in the failing rat hearts was significantly reversed following matrine $(10 \mathrm{mg} / \mathrm{kg})$ treatment. Similarly, matrine also reversed the increase of eNOS protein in the failing hearts $(\mathrm{P}<0.05$; Fig. $5 \mathrm{~B})$. This suggests that the blockage of $\beta 3$-AR/eNOS pathway by matrine also contributes to its protective effects.

\section{Discussion}

In the present study, it was firstly revealed that matrine treatment improved the function and structure of failing rat hearts, and its mechanisms were associated with inhibiting apoptosis and blocking $\beta 3$-AR in cardiomyocytes. These findings implicate matrine as a novel therapeutic drug for heart failure.

Heart failure is a common severe condition that prevents efficient circulation of the blood around the body, and most commonly occurs secondary to myocardial infarction, hypertension, valvular heart disease and cardiomyopathy. Heart failure remains a leading cause of hospitalization in people $>65$ years old and affects $\sim 5$ million Americans, with $\sim 550,000$ people diagnosed annually (17). Therefore, the development of therapeutic drugs to treat heart failure are urgently required.

Matrine is a type of alkaloid extracted from Sophora flavescens AIT, which has been used to treat diseases for centuries (6). Numerous studies have demonstrated that matrine possesses numerous biological activities, including antitumor, antivirus, antioxidant and anti-inflammatory actions $(3,6-8,12)$. However, the effects of matrine on heart failure have not previously been elucidated. Therefore, this study was designed to observe whether matrine may have cardioprotective effects against myocardial failure and to examine its potential mechanisms. As myocardial infarction is a leading cause of heart failure clinically, in the present study a chronic heart failure model was established in rats by coronary artery ligation according to previous studies (15). Consistently, four weeks following surgery, echocardiography examination demonstrated the decline in heart function in rats subjected to myocardial infarction and confirmed the successful establishment of a heart failure model in rats. However, following matrine treatment, the decrease of EF and the increase of LVEDS in CHF rats were evidently attenuated. These results suggest that matrine is able to improve the function of the failing heart. In addition, matrine also inhibited the increase in the levels of serum AST, LDH and CPK. It was also identified that myocardial hypertrophy and cardiac fibrosis were present in the failing heart, and that matrine treatment was able to suppress the increase of heart hypertrophy and cardiac fibrosis. This suggests that matrine alleviates the structural remodeling of the failing heart. Consistent with these results, a previous study also reported that matrine was able to prevent pressure overload-induced myocardial hypertrophy and fibrosis (18). These data suggest that matrine not only prevents cardiac hypertrophy but also rescues heart failure. However, the underlying mechanisms remain unclear.
It is well estabclished that a major molecular characteristic feature of heart failure is loss of cardiac myocytes through apoptosis, which has been demonstrated to be an important contributor to the progression of the condition (19). Recent studies have demonstrated that cardiomyocyte apoptosis also occurs following acute myocardial infarction, as well as in the hypertrophied and the aging heart, which are conditions that are frequently associated with the development of heart failure (19-20). Therefore, the inhibition of apoptosis may prevent the occurrence and development of heart failure. The present study also investigated the effects of matrine on the apoptosis in the myocardium of failing hearts. The results revealed that matrine treatment evidently enhanced the expression of anti-apoptotic protein Bcl-2 and inhibited the augmentation of pro-apoptotic Bax expression in rats with heart failure. The anti-apoptotic actions of matrine contributed, at least in part, to its cardioprotective effects on heart failure.

In the heart, $\beta 3$-AR has an important role in the modulation of cardiac function $(16,21)$. Our previous study demonstrated that $\beta 3$-AR protein was overexpressed in ISO-induced heart failure in rats, and the blockage of $\beta 3$-AR exerted a protective effect on the decline of heart function (16). This suggests that $\beta 3$-AR blockers may be novel therapeutic agents for heart failure $(16,22)$. Similarly, upregulation of the $\beta 3$-AR expression was also observed in the failing canine myocardium and the functional response to $\beta 3$-AR stimulation was increased, which may promote the progression of cardiac dysfunction in chronic heart failure (23). Consistent with this, several recent studies have reported that activation of $\beta 3$-AR inhibited cardiac contraction via the eNOS pathway (21). In the present study, it was observed that matrine is able to attenuate the upregulation of $\beta 3$-AR protein and also decrease the expression of eNOS in failing hearts. Furthermore, the blockade of $\beta 3-\mathrm{AR}$ and nNOS also contributes to the protection from heart failure by matrine.

In conclusion, to the best of our knowledge, this is the first study to demonstrate that matrine has a therapeutic effect on heart failure, and its underling mechanisms are associated with the inhibition of cardiomyocyte apoptosis and the overexpression of $\beta 3-\mathrm{AR}$ and eNOS proteins. These results suggest that matrine may be a potential therapeutic agent for the treatment of heart failure.

\section{Acknowledgements}

This study was supported by the Natural Science Foundation of Heilongjiang Province of China (grant no. D201105 to Professor Runtao Gan), and Science and Technology Fund of Heilongjiang Education Bureau (grant no. 12511297).

\section{References}

1. Ma L, Wen S, Zhan Y, He Y, Liu X and Jiang J: Anticancer effects of the Chinese medicine matrine on murine hepatocellular carcinoma cells. Planta Med 74: 245-251, 2008.

2. Jin JH, Kim JS, Kang SS, Son KH, Chang HW and Kim HP: Anti-inflammatory and anti-arthritic activity of total flavonoids of the roots of Sophora flavescens. J Ethnopharmacol 127: 589-595, 2010.

3. Yan F, Liu Y and Wang W: Matrine inhibited the growth of rat osteosarcoma UMR-108 cells by inducing apoptosis in a mitochondrial-caspase-dependent pathway. Tumour Biol 34: 2135-2140, 2013 
4. Kim DI, Lee TK, Lim IS, Kim H, Lee YC and $\mathrm{Kim} \mathrm{CH}$ Regulation of IGF-I production and proliferation of human leiomyomal smooth muscle cells by Scutellaria barbata D. Don in vitro: isolation of flavonoids of apigenin and luteolin as acting compounds. Toxicol Appl Pharmacol 205: 213-224, 2005.

5. Zhang HF, Shi LJ, Song GY, Cai ZG, Wang C and An RJ: Protective effects of matrine against progression of high-fructose diet-induced steatohepatitis by enhancing antioxidant and anti-inflammatory defences involving Nrf2 translocation. Food Chem Toxicol 55: 70-77, 2013.

6. Tan C, Qian X, Jia R, Wu M and Liang Z: Matrine induction of reactive oxygen species activates p38 leading to caspase-dependent cell apoptosis in non-small cell lung cancer cells. Oncol Rep 30: 2529-2535, 2013.

7. Zhang S, Zhang Y, Zhuang Y, et al: Matrine induces apoptosis in human acute myeloid leukemia cells via the mitochondrial pathway and Akt inactivation. PLoS One 7: e46853, 2012.

8. Li LQ, Li XL, Wang L, et al: Matrine inhibits breast cancer growth via miR-21/PTEN/Akt pathway in MCF-7 cells. Cell Physiol Biochem 30: 631-641, 2012.

9. Haiyan W, Yuxiang L, Linglu D, et al: Antinociceptive effects of matrine on neuropathic pain induced by chronic constriction injury. Pharm Biol 51: 844-850, 2013.

10. Li H, Huang D, Gao Z, et al: Scutellarin inhibits cell migration by regulating production of alphavbeta6 integrin and E-cadherin in human tongue cancer cells. Oncol Rep 24: 1153-1160, 2010.

11. Zhou Y, Shan H, Qiao G, Sui X, Lu Y and Yang B: Inotropic effects and mechanisms of matrine, a main alkaloid from Sophora flavescens AIT. Biol Pharm Bull 31: 2057-2062, 2008.

12. Zhou Y, Xu W, Han R, et al: Matrine inhibits pacing induced atrial fibrillation by modulating $\mathrm{I}(\mathrm{KM} 3)$ and $\mathrm{I}(\mathrm{Ca}-\mathrm{L})$. Int $\mathrm{J}$ Biol Sci 8: 150-158, 2012.

13. Ai J, Gao HH, He SZ, Wang L, Luo DL and Yang BF: Effects of matrine, artemisinin, tetrandrine on cytosolic $\left[\mathrm{Ca}^{2+}\right] \mathrm{i}$ in guinea pig ventricular myocytes. Acta Pharmacol Sin 22: 512-515, 2001.
14. Ren XP, Wu J, Wang X, et al: MicroRNA-320 is involved in the regulation of cardiac ischemia/reperfusion injury by targeting heat-shock protein 20. Circulation 119: 2357-2366, 2009.

15. Loot AE, Roks AJ, Henning RH, et al: Angiotensin-(1-7) attenuates the development of heart failure after myocardial infarction in rats. Circulation 105: 1548-1550, 2002.

16. Gan RT, Li WM, Xiu CH, et al: Chronic blocking of beta 3-adrenoceptor ameliorates cardiac function in rat model of heart failure. Chin Med J (Engl) 120: 2250-2255, 2007.

17. Schleithoff SS, Zittermann A, Tenderich G, Berthold HK, Stehle P and Koerfer R: Vitamin D supplementation improves cytokine profiles in patients with congestive heart failure: a double-blind, randomized, placebo-controlled trial. Am J Clin Nutr 83: 754-759, 2006

18. Zhang YJ, Xiang MX, San J, Cheng G and Wang SS: Effect of matrine and carvedilol on collagen and MMPs activity of hypertrophy myocardium induced by pressure overload. J Zhejiang Univ Sci B 7: 245-250, 2006

19. Sabbah HN and Sharov VG: Apoptosis in heart failure. Prog Cardiovasc Dis 40: 549-562, 1998.

20. van Empel VP, Bertrand AT, Hofstra L, Crijns HJ, Doevendans PA and De Windt LJ: Myocyte apoptosis in heart failure. Cardiovasc Res 67: 21-29, 2005

21. Moens AL, Yang R, Watts VL and Barouch LA: Beta 3-adrenoreceptor regulation of nitric oxide in the cardiovascular system. J Mol Cell Cardiol 48: 1088-1095, 2010.

22. Dessy C and Balligand JL: Beta3-adrenergic receptors in cardiac and vascular tissues emerging concepts and therapeutic perspectives. Adv Pharmacol 59: 135-163, 2010.

23. Cheng HJ, Zhang ZS, Onishi K, Ukai T, Sane DC and Cheng CP: Upregulation of functional beta(3)-adrenergic receptor in the failing canine myocardium. Circ Res 89: 599-606, 2001. 